\title{
HeilberufeSCIENCE auf springerlink.com
}

\author{
Jörg Klewer
}

Online publiziert: 12. Februar 2014

(C) Springer-Verlag Wien 2014

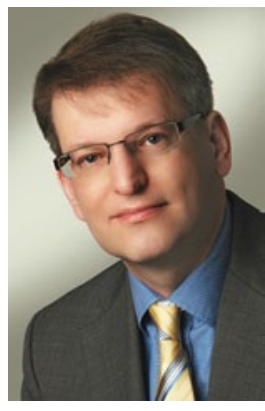

Jörg Klewer

Das Portal springerlink.com bietet neben dem Zugriff auf „online journals“ auch weitere Informationen rund um das jeweilige Journal. In unregelmäßigen Abständen stehen immer wieder andere einzelne Beiträge aus bisherigen HeilberufeSCIENCE-Ausgaben auf der Website von HeilberufeSCIENCE im Portal springerlink.com zum kostenlosen Download zur Verfügung. Somit besteht die Möglichkeit, einen Eindruck von den in HeilberufeSCIENCE veröffentlichten Inhalten und der fachlichen Qualität zu erlangen. Welche Beiträge von den Lesern der Zeitschrift HeilberufeSCIENCE am liebsten abgerufen werden, kann ebenfalls auf der Website von HeilberufeSCIENCE erfahren werden. Deshalb lohnt sich immer wieder zwischen den Erscheinungsterminen von HeilberufeSCIENCE ein Blick auf die Seiten in springerlink.com - auch um dort die neuesten „Online-first“-Publikationen zu finden.

Nun aber liegt eine neue Ausgabe von HeilberufeSCIENCE vor, die sich aus 3 Beiträgen und 2 wissen-

J. Klewer $(\bowtie)$

Zwickau, Deutschland

E-Mail: joklewer@t-online.de schaftlichen Kurzmitteilungen zusammensetzt. Den Anfang macht der international ausgerichtete Beitrag von Janich et al. mit einer epidemiologischen Querschnittsstudie zum Ernährungszustand von Kindern in Nepal. Daran schließt sich der methodisch ausgerichtete Beitrag von Teubner-Guerra und Ochsmann über die Entwicklung und Evaluation eines Fragebogens zur Beurteilung von pathologischem Altruismus an. Die Publikation von Hiemetzberger et al. stellt die Ergebnisse einer Interventionsstudie zu den Effekten von „therapeutic touch“ als ein ganzheitliches Pflegekonzept bei Patienten mit Hörsturz dar.

Die erste wissenschaftliche Kurzmitteilung von Behrendt et al. bildet in einer prägnanten Übersichtsarbeit den Diskussions- und Erkenntnisstand zur Personalbemessung im Pflegedienst deutscher Krankenhäuser ab und kommt zu der Erkenntnis, dass bislang nur wenige am Bedarf ausgerichtete empirisch gesicherte Lösungsansätze existieren. Die 2. wissenschaftliche Kurzmitteilung von Lezock et al. untersucht für das wenig wissenschaftlich untersuchte Arbeitsfeld der ambulanten Pflegedienste den Einsatz eines Verfahrens zur Analyse der Touren- und Einsatzplanung.

An dieser Stelle möchte ich Sie wieder einladen, die 3 Originalarbeiten und die beiden wissenschaftlichen Kurzmitteilungen dieser Ausgabe von HeilberufeSCIENCE kritisch zu lesen und in den wissenschaftlichen Dialog einzutreten - und natürlich würde es mich freuen, wenn ich Sie in der Mai-Ausgabe von HeilberufeSCIENCE im Frühjahr wieder an dieser Stelle begrüßen darf.

Ihr

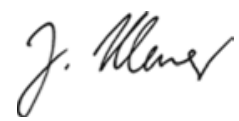

